\title{
Study of the thermal release behaviour of mercury and thallium from liquid eutectic lead-bismuth alloy
}

\author{
By J. Neuhausen* and B. Eichler \\ Laboratory for Radio- and Environmental Chemistry, Paul Scherrer Institute, CH-5232 Villigen PSI, Switzerland
}

(Received May 5, 2004; accepted in revised form October 13, 2004)

Release / Pb-Bi-Eutecticum / LBE / Evaporation / Mercury / Thallium

\begin{abstract}
Summary. The release of mercury and thallium from liquid eutectic lead-bismuth alloy (LBE) under a flowing $\mathrm{Ar} / 7 \%-\mathrm{H}_{2}$ atmosphere has been studied in the temperature range from 408 to $1292 \mathrm{~K}$ using $\gamma$-ray spectroscopy. For technical applications such as liquid metal spallation targets or accelerator driven systems, where liquid LBE is planned to be used as target material, the release of radioactive mercury isotopes produced by spallation is expected to be one of the major safety issues.

During short-term experiments significant amounts of mercury begin to evaporate from liquid $\mathrm{LBE}$ at temperatures starting from about $475 \mathrm{~K} .80 \%$ of the mercury present in the sample is released from samples of approximately $1.5-3 \mathrm{~g}$ within one hour at temperatures higher than $625 \mathrm{~K}$. Thallium release in the temperature range investigated is below experimental error. Long-term experiments reveal that even at temperatures as low as $476 \mathrm{~K}$ about $25 \%$ of the mercury present in the samples is released per day under a flowing $\mathrm{Ar} / 7 \%-\mathrm{H}_{2}$ atmosphere.
\end{abstract}

\section{Introduction}

A eutectic mixture of lead and bismuth (LBE) is planned for use as a target material in liquid metal spallation targets [1] as well as in accelerator driven systems (ADS) for the transmutation of long-lived nuclear waste [2]. In these systems mercury and thallium will be formed by spallation reactions. According to theoretical predictions, approximately $17 \mathrm{~g}$ of mercury and $7 \mathrm{~g}$ of thallium would be produced [3] within 1 year of operation when employing a proton beam current of $1 \mathrm{~mA}$. The chemical behaviour of these elements in liquid LBE is of importance with respect to the safe operation and post-irradiation handling of the target systems and materials as well as for an assessment of the potential risk under various accident scenarios. The evaporation of volatile elements from the target melt is of particular significance. Because in spallation targets and ADS, hydrogen will be formed by spallation, the influence of hydrogen in the cover gas on the release process is of interest.

Investigations on the release of mercury and thallium from liquid metals are scarce. The production of mercury

\footnotetext{
*Author for correspondence (E-mail: joerg.neuhausen@psi.ch).
}

isotopes in liquid lead and bismuth ISOL targets has been studied $[4,5]$. The vaporization of mercury from molten lead under argon and air atmospheres has been investigated in context of the accelerator production of tritium project (APT) [6]. No experimental investigations on the release of mercury and thallium from liquid LBE have been performed to date. Thermochemical properties influencing the evaporation behaviour of volatile nuclear reaction products from liquid metals have been evaluated in [7]. According to the results of this evaluation, mercury is expected to be easily volatilised from liquid lead and bismuth. The release of thallium may also be possible, but much smaller evaporation rates should be expected. Because the chemical interaction of mercury and thallium with lead, bismuth and LBE is expected to be very similar, the results of [7] should be applicable to LBE as well. To confirm these results experimentally, we studied the thermal release behaviour of mercury and thallium from liquid LBE under an $\mathrm{Ar} / 7 \%-\mathrm{H}_{2}$-atmosphere.

\section{Experimental}

LBE samples (44.8 wt. \% Pb, 55.2 wt. \% Bi) were prepared by melting the appropriate amounts of $\mathrm{Pb}$ granules (Alfa Aesar, 99.9999\%) and Bi pieces (Berg- und Hüttenkombinat "Albert Funk", Freiberg/Sachsen, Germany, 99.9999\%) at $773 \mathrm{~K}$ for $1 \mathrm{~h}$ under an $\mathrm{Ar} / 7 \% \mathrm{H}_{2}$-atmosphere. The samples were then activated by neutron irradiation at the Swiss spallation neutron source SINQ, where mercury and thallium nuclides were formed by $(n, x n)$ and $(n, p x n)$ reactions on the sample materials and subsequent electron capture or $\beta^{+}$decay of $\mathrm{Pb}$ and $\mathrm{Bi}$ nuclides. Among these nuclides no mercury isotope could be found with suitable decay properties for our experiments, i.e. one with $\gamma$-ray energies not overlapping with the $\gamma$-ray energies of $\mathrm{Pb}$ and $\mathrm{Bi}$ nuclides present in the samples and also a suitable half-life for experiments on a time scale ranging from an hour to several days. Therefore, ${ }^{203} \mathrm{Hg}\left(t_{1 / 2}=46.61 \mathrm{~d}[8]\right)$, which is produced directly by $(n, \alpha)$ reaction of ${ }^{206} \mathrm{~Pb}$, was chosen for the study of mercury release. Because its lone $\gamma$-ray signal at $279.20 \mathrm{keV}$ coincides with a signal from ${ }^{203} \mathrm{~Pb}\left(t_{1 / 2}=51.873 \mathrm{~h}\right.$ [8] $)$, the samples were allowed to decay for $4-5$ weeks to ensure the decay of ${ }^{203} \mathrm{~Pb}$ before mercury release experiments were started. ${ }^{203} \mathrm{~Pb}$ decay was monitored by $\gamma$-ray spectroscopy. 
For the evaluation of thallium release the $439.56 \mathrm{keV} \gamma$-ray signal of ${ }^{202} \mathrm{Tl}\left(t_{1 / 2}=12.23 \mathrm{~d}[8]\right)$ was used.

Sample masses were $1.4-2.8 \mathrm{~g}$ for short-term and $6.7 \mathrm{~g}$ for long-term experiments. The abundances of nuclei and concentrations of ${ }^{203} \mathrm{Hg}$ and ${ }^{202} \mathrm{Tl}$ in the samples were determined from the peak areas of the above-mentioned characteristic $\gamma$-rays of the respective nuclide, taking into account the detector efficiency and $\gamma$-branching [8]. Self-absorption effects were roughly estimated based on sample thickness and mass attenuation coefficients listed in [9]. Typical abundances of nuclei at the outset of short-term release experiments were in the range of $5 \times 10^{8}$ to $1 \times 10^{9}$ for ${ }^{203} \mathrm{Hg}$ and $8 \times 10^{8}$ to $2 \times 10^{9}$ for ${ }^{202} \mathrm{Tl}$. The corresponding mole fractions were in the range of $9 \times 10^{-14}$ to $2 \times 10^{-13}$ for ${ }^{203} \mathrm{Hg}$ and 2 to $3 \times 10^{-13}$ for ${ }^{202} \mathrm{Tl}$. For the two long-term experiments at 476 and $515 \mathrm{~K}$ samples with abundances of nuclei of $4 \times 10^{9}$ and $1 \times 10^{9}\left({ }^{203} \mathrm{Hg}\right)$ as well as $3 \times 10^{8}$ and $2 \times 10^{8}$ $\left({ }^{202} \mathrm{Tl}\right)$ were used, respectively. Mole fractions for these samples were $2 \times 10^{-13}$ and $5 \times 10^{-14}\left({ }^{203} \mathrm{Hg}\right)$ and $2 \times 10^{-14}$ and $1 \times 10^{-14}\left({ }^{202} \mathrm{Tl}\right)$, respectively.

Evaporation experiments (one experiment for each temperature setting) were performed using the experimental setup illustrated in Fig. 1. The samples were scratched to remove the surface oxide layer and placed on a quartz tissue within a quartz boat, which was furthermore placed in a quartz tube. This tube was flushed with an $\mathrm{Ar} / 7 \%-\mathrm{H}_{2}$ mixture (purity: $\mathrm{H}_{2}>99.993 \%, \mathrm{Ar}>99.998 \%$ ) which was previously forced through a column containing a Pd-catalyst (to facilitate the establishment of $\mathrm{O}_{2} / \mathrm{H}_{2} / \mathrm{H}_{2} \mathrm{O}$ equilibrium) and Sicapent (with indicator, Merck, Germany) for removing moisture. A water partial pressure of $3.7 \pm 1.7 \mathrm{hPa}$ was determined using a $\mathrm{Zr} / \mathrm{Y}_{2} \mathrm{O}_{3}$-solid electrolyte cell. This value corresponds to an oxygen partial pressure expected for a theoretical $\mathrm{PbO}$ equilibrium activity of $10^{-5}$ within the molten alloy. All experiments were performed using a continuous gas flow of $60 \mathrm{ml} / \mathrm{min}$ adjusted by a mass flow controller. The apparatus was flushed for approximately $20 \mathrm{~min}$ after the insertion of the sample to remove air contamination. The tube was resistance-heated to the desired temperatures. Temperatures were measured and controlled using thermocouples and a thyristor controller. Two charcoal filters were placed at the end of the tube to prevent volatile radioactive species from reaching the exhaust.

$\gamma$-ray spectroscopic measurements were performed using an EG\&G ORTEC GEM-13180 coaxial HPGe-detector equipped with standard Canberra electronics (4k multichannel analyser). The detector was shielded with lead bricks of
$5 \mathrm{~cm}$ thickness to suppress background radiation. The data were recorded and processed using the Canberra Genie 2000 software on a standard PC.

\section{Short-term experiments}

A $\gamma$-ray spectrum of the sample was recorded before each heating experiment (measuring time $1 \mathrm{~h}$ ). The sample was then placed into the evaporation apparatus, which was flushed with the $\mathrm{Ar} / 7 \%-\mathrm{H}_{2}$ mixture. After approximately $20 \mathrm{~min}$, the apparatus was heated to the desired temperature within $10 \mathrm{~min}$ and then held at this temperature for $50 \mathrm{~min}$. The sample was then cooled to room temperature within $10 \mathrm{~min}$ using a fan. An additional $\gamma$-ray spectrum was collected after the heating experiment (measuring time $1 \mathrm{~h}$ ). The fractional release of mercury and thallium was calculated from the integrated peak areas of the abovementioned characteristic $\gamma$-rays of ${ }^{203} \mathrm{Hg}$ and ${ }^{202} \mathrm{Tl}$ before and after heating according to Eq. (1). Decay corrections were omitted given the limited duration of the experiment compared to the long half-lives of the nuclides studied. ${ }^{206} \mathrm{Bi}$ $\left(t_{1 / 2}=6.24 \mathrm{~d}[8]\right)$, which is formed by $(n, x n)$ reactions during neutron irradiation, was used as an internal standard to correct for geometry and self-absorption changes that occurred between the measurements before and after heating due to the melting process. The $\gamma$-ray signal at $343.51 \mathrm{keV}$ of ${ }^{206} \mathrm{Bi}$ [8] was used for these corrections. Typical corrections amounted to $10 \%-30 \%$, reflecting substantial changes of the sample shape upon melting. No measurable evaporation of $\mathrm{Bi}$ was detected at the temperatures investigated.

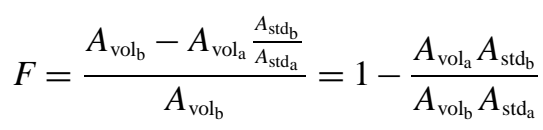

$F$ : fractional release;

$A_{\text {vol }}$ : peak area of $\gamma$-ray signal of the volatile $\left({ }^{203} \mathrm{Hg}\right.$ or $\left.{ }^{202} \mathrm{Tl}\right)$ before heating;

$A_{\text {vola }_{3}}$ : peak area of $\gamma$-ray signal of the volatile $\left({ }^{203} \mathrm{Hg}\right.$ or $\left.{ }^{202} \mathrm{Tl}\right)$ after heating;

$A_{\text {std }}$ : peak area of $\gamma$-ray signal of the ${ }^{206} \mathrm{Bi}$ standard before heating;

$A_{\text {std }}$ : peak area of $\gamma$-ray signal of the ${ }^{206} \mathrm{Bi}$ standard after heating.

Errors indicated by error bars in the figures were estimated from the expression

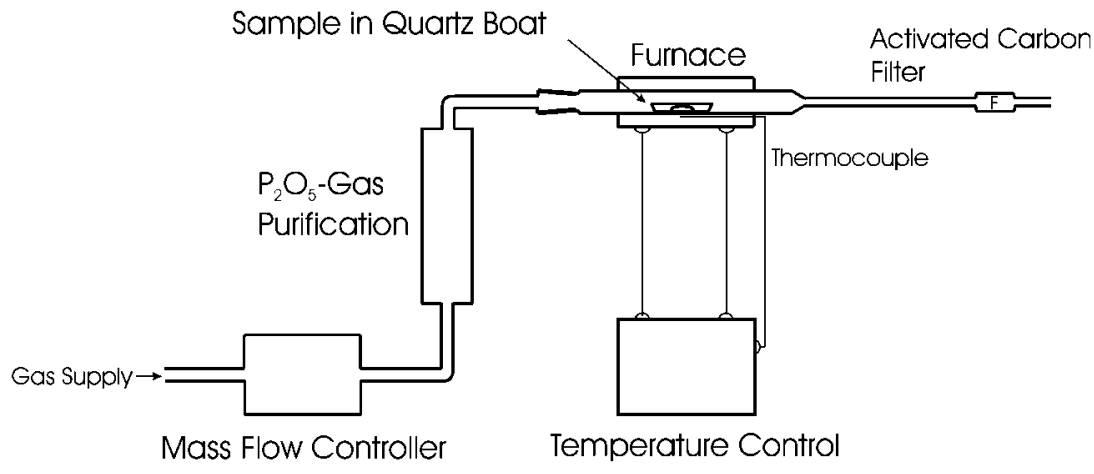

Fig. 1. Schematic drawing of the experimental apparatus. 


$$
\begin{aligned}
\Delta F= & \left|\frac{\delta F}{\delta A_{\mathrm{vol}_{\mathrm{b}}}} \Delta A_{\mathrm{vol}_{\mathrm{b}}}\right|+\left|\frac{\delta F}{\delta A_{\mathrm{vol}_{\mathrm{a}}}} \Delta A_{\mathrm{vol}_{\mathrm{a}}}\right| \\
& +\left|\frac{\delta F}{\delta A_{\mathrm{std}_{\mathrm{b}}}} \Delta A_{\mathrm{std}_{\mathrm{b}}}\right|+\left|\frac{\delta F}{\delta A_{\mathrm{std}_{\mathrm{a}}}} \Delta A_{\mathrm{std}_{\mathrm{a}}}\right| .
\end{aligned}
$$

Values for $\Delta A$ were derived from $1 \sigma$ errors evaluated using the peak fit routine of the Genie 2000 software.

\section{Long-term experiments}

The experimental set-up used in the short-term experiments was placed on the $\gamma$-ray detector which was cooled by circulating water. $\gamma$-ray spectra were taken continuously for periods up to 7 days using a batch routine. Measuring times were $2 \mathrm{~h}$ for each spectrum. A decay correction was applied to the integrated peak areas of the $\gamma$-ray signals of ${ }^{203} \mathrm{Hg}$, ${ }^{202} \mathrm{Tl}$ and the ${ }^{206} \mathrm{Bi}$-standard. Errors in measuring times and half-lives of the investigated nuclides were neglected in the error calculations because they are at least one order of magnitude smaller than the errors resulting from counting statistics. Maximum corrections for self-absorption and geometry changes amounted to $8 \%$.

\section{Results and discussion}

Fig. 2 shows the results of short-term $(1 \mathrm{~h})$ evaporation experiments at various temperatures between 408 and $1292 \mathrm{~K}$. Measurable amounts of mercury began to evaporate from liquid LBE under a flowing $\mathrm{Ar} / 7 \%-\mathrm{H}_{2}$ atmosphere at temperatures around $475 \mathrm{~K}$. At $593 \mathrm{~K}$ about $50 \%$ of the mercury present in our samples was released within $1 \mathrm{~h}$. At temperatures higher than $625 \mathrm{~K}$, most of the mercury present in the samples $(80 \%)$ was evaporated. Hence, for a liquid LBE spallation target (operating temperatures 473-723 K) substantial evaporation of the mercury isotopes produced by spallation can be expected in case of continuous pumping of the cover gas or a leakage. No thallium release was observed within the limits indicated by error bars under our experimental conditions for temperatures up to $1292 \mathrm{~K}$. This finding is consistent with thermochemical evaluations [7], because according to these studies, thallium should be only slightly more volatile than the target materials themselves.

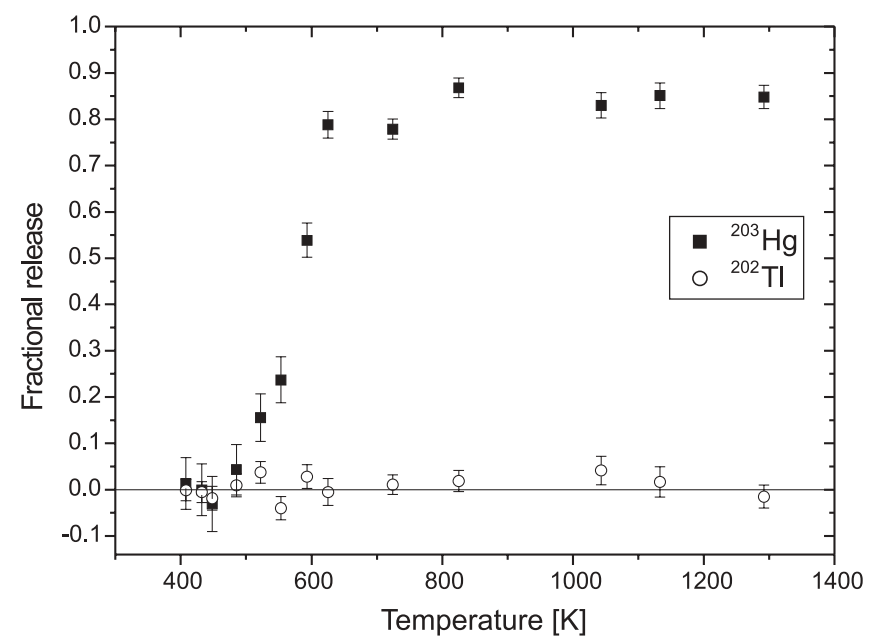

Fig. 2. Fractional release of mercury and thallium from LBE ( $1 \mathrm{~h} \mathrm{ex-}$ periments) in $\mathrm{Ar} / 7 \%-\mathrm{H}_{2}$ atmosphere as a function of temperature.
Considering the low concentrations of thallium present in the samples and the resulting low partial pressure of thallium over the samples, we would not have expected the release of significant amounts of thallium.

The results of long-term experiments are presented in Fig. 3. Here the fractional release of mercury and thallium from LBE measured in an $\mathrm{Ar} / 7 \%-\mathrm{H}_{2}$ atmosphere at 476 and $515 \mathrm{~K}$ is shown as a function of the square root of heating time for periods up to 7 days. At $476 \mathrm{~K}$, which can be considered as a lower limit for the operation of a liquid LBE spallation target (standby temperature), about $25 \%$ of the mercury present in the sample was released within the first day of the experiment. At $515 \mathrm{~K}$ about $60 \%$ was released within the first day, while a fractional release of over $90 \%$ was observed within one week. The fractional release shows an approximately linear relationship to the square root of heating time for release fractions up to $45 \%$. This behaviour is generally known for release processes [10]. No significant amounts of thallium were released in these long-term experiments within the limits indicated by error bars in the figures.

Experiments dealing with the evaporation of mercury from molten lead are described in [6]. These studies were performed at a single temperature $(613 \mathrm{~K})$ using much higher concentrations of mercury $\left(x \approx 10^{-4}\right.$ to $\left.10^{-3}\right)$, a much larger sample $(2.5 \mathrm{~kg} \mathrm{~Pb})$ and different gas atmospheres (high purity Ar, air). The influences of agitation of the melt as well as the addition of aluminium as an impurity on the evaporation behaviour of mercury were investigated by these authors. In comparison to these experiments, we generally observed much larger values for the fractional release of mercury: at $613 \mathrm{~K}$ under a high purity argon atmosphere a fractional release of mercury of 10.2 and $23.4 \%$ within one week for mercury mole fractions of $10^{-4}$ and $10^{-3}$, respectively, were reported in [6]. In our experiments we found a fractional release of $54 \%$ of the mercury present in our samples within one hour at $593 \mathrm{~K}$. This difference may be explained by the much larger samples used in [6] which led to longer transport times for mercury to reach the sample surface. Another reason may be the presence/absence of an oxide layer on the sample surface. In [6] the formation of such an oxide layer on the $\mathrm{Pb}$ melt under an air atmosphere led to a decrease of the

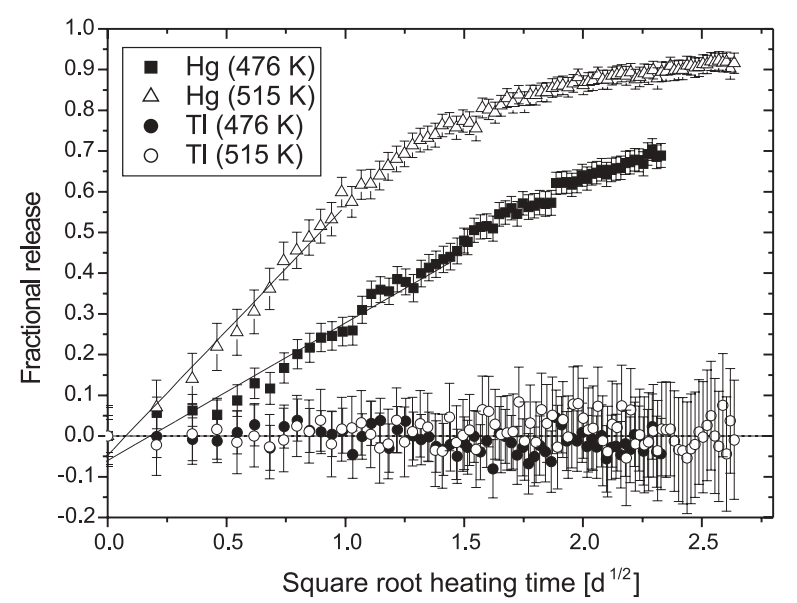

Fig. 3. Comparison of the long-term mercury and thallium release from LBE in an $\mathrm{Ar} / 7 \%-\mathrm{H}_{2}$ atmosphere at different temperatures as a function of the square root of heating time. 
release rate of mercury by an order of magnitude compared to experiments performed with high purity argon as a cover gas. In our experiments using $\mathrm{Ar} / 7 \%-\mathrm{H}_{2}$ as a cover gas, a thin oxide layer, which might be present even under a high purity Ar atmosphere (resulting from lead oxides present in the starting material), will be reduced by hydrogen, thus giving rise to a higher evaporation rate. From an evaluation of the chemical interaction of mercury with either lead or bismuth using Miedema's macroscopic atom model $[7,11]$ it seems unlikely that the difference in evaporation rates would have been caused by much stronger chemical interactions of mercury with lead compared to bismuth or LBE. The partial molar enthalpies of solution for mercury in lead and bismuth were calculated to be +5.0 and $+4.3 \mathrm{~kJ} / \mathrm{mol}$, respectively $[7,11]$. Assuming close to ideal behaviour for the eutectic mixture, the chemical interaction of mercury with LBE should be comparable to the interaction with the pure components of the melt.

From a physicochemical point of view, thermal release can be regarded as an activated process involving different steps. For the simplest case of monoatomic species, diffusion of the volatile species onto the surface and subsequent desorption and evaporation have to be taken into account. In case of the presence of a solid surface oxide layer, the diffusion of the volatile species will be additionally retarded. From an estimation of the activation energy of the release process it may be possible to identify the step that determines the rate of evaporation. An evaluation of thermodynamic data of mercury and thallium oxides [12] reveals that both elements should be released in the elemental form under the reducing conditions present in our experiments. The description of the temperature dependency of mercury release using an Arrhenius-type relation results in an activation energy of $54 \mathrm{~kJ} / \mathrm{mol}$ for this process. This value compares well with the enthalpy of evaporation of mercury from liquid lead $(56.4 \mathrm{~kJ} / \mathrm{mol})$ and bismuth $(57.1 \mathrm{~kJ} / \mathrm{mol})$ calculated in [7], which can be regarded as a lower limit for the activation energy of the desorption/evaporation process. Experimental values determined for the activation energies for self-diffusion in liquid-lead bismuth mixtures range from 11.6 to $40.5 \mathrm{~kJ} / \mathrm{mol}$ [13]. No data are available for the diffusion of mercury in either liquid lead or bismuth. However, activation energies for diffusion of the mercury homologues zinc and cadmium in liquid lead and bismuth range from $13.4-23.4 \mathrm{~kJ} / \mathrm{mol}$ [13]. From these considerations it seems likely that the process of desorption/evaporation determines the rate of mercury release from liquid LBE rather than diffusion in our experiments using small samples. However, the fact that the release rate of mercury from liquid lead observed in [6] is much smaller and strongly influenced by stirring the sample indicates that transport within the liquid becomes more important in case of larger samples. A quantitative description of diffusion and desorption kinetics is beyond the scope of our experiments.

With respect to technical applications of liquid LBE such as spallation targets and ADS the following conclusions can be drawn:

From the results presented in this work, the release of significant amounts of mercury has to be expected for a liquid LBE spallation target system or an LBE based ADS. This particularly applies to accident scenarios where volatiles can escape from the broken system before LBE solidifies, or if continuous pumping of the cover gas is considered. For a closed system the amount of mercury present in the cover gas will be limited by the equilibrium vapour pressure of mercury over the highly diluted solution of mercury in LBE ( $\mathrm{Hg}$ mole fractions around $\left.10^{-5}\right)$. Considering the scenario of continuous pumping, the release of mercury in a large target with a much smaller surface/volume ratio will be retarded due to longer transport times within the liquid, compared to the small samples used in this work. However, within a liquid metal target, convection will increase the release rate [6]. Furthermore, in a liquid metal target spallation products will be formed that can react with the oxide layers of construction materials to form oxides that are not reducible by hydrogen. If these oxides accumulate at the LBE surface, the release of volatiles can be retarded as compared to our results. To elucidate these influences, larger scale experiments are planned, involving variations of sample size, concentration of volatiles, gas flow, temperature and flow conditions of the liquid, as well as the addition of representative elements for spallation products. The release of significant amounts of thallium from a liquid LBE spallation target is not to be expected according to the results presented above.

Acknowledgment. This work was supported by the BBW under Contract Nr. 01.0298 "Megapie-Test", EU Contract No. FIKW-CT-200100159 and the EU-RTD project TARGISOL (HPRI-CT-2001-50033). Furthermore, we thank Mr. H.-U. Aebersold for neutron activation of LBE samples and Dr. R. Dressler for assistance in $\gamma$-ray spectroscopy.

\section{References}

1. Salvatores, M., Bauer, G. S., Heusener, G.: The MEGAPIE Initiative. PSI-Report Nr. 00-05, Paul Scherrer Institut, Villigen, Switzerland (2000).

2. Gromov, B. F., Belomitlev, Yu. S., Efimov, E. I., Leonchuk, M. P., Martinov, P. N. Orlov, Yu. I., Pankratov, D. V., Pashkin, Yu. G., Toshinsky, G. I., Chenukov, V. V., Shmatko, B. A., Stepankov, V.S.: Use of lead-bismuth coolant in nuclear reactors and accelerator-driven systems. Nucl. Engin. Design 173, 207 (1997).

3. Atchison, F.: Nuclide Production in the SINQ Target. Report SINQ/816/AFN-702, Paul Scherrer Institut, Villigen, Switzerland (1987).

4. Ravn, H. L., Sundell, S., Westgaard, L.: Target techniques for the ISOLDE on-line separator. Nucl. Instrum. Methods 123, 131 (1975).

5. Köster, U.: For the ISOLDE Collaboration: ISOLDE target and ion source chemistry. Radiochim. Acta 89, 749 (2001).

6. http://www.dne.bnl.gov/atd-mag/htm/ HgVaporizationJournal.PDF.

7. Eichler, B., Dressler, R.: Verflüchtigung von Kernreaktionsprodukten aus geschmolzenen Metalltargets. PSI-Report 95-03, Paul Scherrer Institut, Villigen, Switzerland (1995).

8. http://nucleardata.nuclear.lu.se/nucleardata/toi/.

9. http://physics.nist.gov/PhysRefData/XrayMassCoef/tab3.html.

10. Inthoff, W., Zimen, K. E.: Kinetik der Diffusion radioaktiver Edelgase aus festen Stoffen nach Bestrahlung (Edelgasdiffusion in Festkörpern 2). Trans. Chalmers Univ. Technol., Gothenburg, Nr. 196 (1956).

11. Niessen, A. K., de Boer, F. R., Boom, R., de Châtel, P. F., Mattens, W. C. M.: Model predictions for the enthalpy of formation of transition metal alloys II. CALPHAD 7, 51 (1983).

12. Barin, I.: Thermochemical Data of Pure Substances. VCH, Weinheim (1995).

13. Landolt-Börnstein: Zahlenwerte und Funktionen aus Physik, Chemie, Astronomie, Geophysik und Technik. 6. Auflage, II. Band, 5. Teil B, Springer, Berlin (1968). 